\title{
Resistance training is an effective exercise therapy in cardiac rehabilitation program for patients with coronary artery disease: a systematic review
}

\author{
Salwa B. El-Sobkey ${ }^{*}$ (1)
}

\begin{abstract}
Background: Exercise-based cardiac rehabilitation program is a comprehensive intervention for the rehabilitation of coronary artery disease patients. Exercise therapy is a valuable, effective, and integral intervention of cardiac rehabilitation. Aerobic training is the gold standard exercise therapy in cardiac rehabilitation programs. In addition, combined training with added resistant training is also used. This systematic review aimed to provide the physical therapy clinicians with a consensus regarding the effect of resistant training by answering the question of what is the cardiovascular and non-cardiovascular effect (s) of resistant training in the cardiac rehabilitation program for patients with coronary artery disease?

Main body: The Physiotherapy evidence database (PEDro) was used as a search engine to select articles through study eligibility criteria. Adult or adult and old stable coronary artery disease patients engaged in a site-based (Phase II) exercise-based cardiac rehabilitation program that includes aerobic and resistant training. Selected articles applied cardiovascular and non-cardiovascular outcome measures to measure the effectiveness of resistant training. The author reviewed the selected articles and applied quantitative non-statistical analysis and appraisal for these articles. The systematic selection process resulted in 10 studies with a total participants number of 3877 . Analyzing the articles revealed that adding resistant training to aerobic training resulted in a favorable improvement in outcome measures for coronary artery disease patients. Resistant training produced improvement in the exercise capacity $\left(\mathrm{VO}_{2}\right.$ peak), blood pressure, skeletal muscle strength, endurance, body composition, sleep quality, depression, and health-related quality of life.
\end{abstract}

Short conclusions: The addition of resistant training to aerobic training in cardiac rehabilitation induces more positive cardiovascular and non-cardiovascular effects for stable coronary artery disease patients.

Keywords: Cardiac rehabilitation, Coronary artery disease, Aerobic training, Resistance training, Exercise therapy, Skeletal muscle strength and endurance, Quality of life, Body composition, Depression, Sleep quality

*Correspondence: salwael-sobkey@pt.bsu.edu.eg

Faculty of Physical Therapy, Beni-Suef University, Beni-Suef, Egypt

\section{Background}

Cardiac rehabilitation program (CRP) is a comprehensive useful and effective program for prevention and rehabilitation which is recommended by the American Heart Association and the American College of Cardiology for treatment and management of patients with coronary artery disease (CAD) [1-7]. Exercise therapy 
is an integral component of CRP proved to be a valuable and effective non-pharmacological intervention for patients with CAD and is designed to improve exercise capacity, cardiovascular health, and overall health status [1, 2, 8-11]. The American Heart Association guideline recommends aerobic training (AT) and resistance training (RT) as the core elements of exercise-based CRP [3]. Traditionally in CRP, the AT has been considered as the basic exercise modality which is proved to be effective in improving and maintaining exercise capacity and cardiovascular fitness [12-15]. In addition to AT and RT, the exercise-based CRP consists of combined training (CT) in which the RT is added to the AT and showed to be an effective intervention to restore physical function and exercise capacity and to improve coronary risk factors including hypertension, hyperglycemia, and dyslipidemia in CAD patients [2]. The addition of RT to AT in CRP enhances strength and endurance of skeletal muscles (SM), functional capacity and level of independence, health-related quality of life (HRQOL), as well as reduces disability [16-19]. The effects of RT also include a positive impact on metabolic risk factors, functional capacity, and psychosocial well-being on CAD patients [16, 20]. However, there is a lack of clear consensus regarding the exact effects of RT as a part of CRP for CAD patients [1]. And although the safety and efficacy of RT for patients with CAD [21-24], both post-myocardial infarction (MI) $[25,26]$ and post coronary artery bypass graft (CABG) surgery [27], has been recently investigated and demonstrated beneficial effects, there is insufficient information available and there is still a need to draw a conclusion [17, $18,28-32]$. It is also believed that because RT is important in CRP, additional research is required to determine the effectiveness of RT as added to AT [33]. Complications of CRP in general and exercise therapy specific for CAD patients include major adverse cardiovascular events and even death [34]. These serious complications raise the importance of depending on evidence-based practice for the implementation of exercise-based CRP for CAD patients. The Preferred Reporting Items for Systematic Reviews and Meta-Analyses (PRISMA) is an evidence-based minimum set of items for reporting in systematic reviews and meta-analyses. The PRISMA focuses on the reporting of reviews evaluating randomized trials but can also be used as a basis for reporting systematic reviews of other types of research, particularly evaluations of interventions [35]. Therefore, the purpose of the current systematic review is to provide the physical therapy clinicians with a consensus regarding the effects of RT by answering the question of what is the cardiovascular and non-cardiovascular effect (s) of resistant training in the cardiac rehabilitation programs for stable CAD patients?

\section{Main text}

The study protocol and information source used the PRISMA items, including the PRISMA 2009 checklist, were the reference of the current systematic review reporting [35] and the physiotherapy evidence database (PEDro) was the search engine for it. The PEDro is a free database of over 43,000 randomized trials, systematic reviews, and clinical practice guidelines in physiotherapy, and all trials on PEDro are independently assessed for quality [36]. Eligibility Criteria: The following inclusion criteria were considered for records (articles/studies) selection: 1- Type of records: randomized controlled trials (RCTs), systemic review (SR), and meta-analysis (MA) 2- Time frame: records published within the last 10 years (2010 to 2019), 3Age of participants: Adult (18-60 years) or adult and old (above 60 years). 4- Diagnosis: patients with stable CAD (e.g., MI, CABG surgery), 5- Type of CRP: Sitebased supervised CRP (Phase II cardiac rehabilitation, 5-9 days post-cardiac event or cardiac revascularization), 6- Objective of the records: to measure the effects of RT, 7- Language of the records: English language, 8- Type of intervention: RT or CT, 9- Type of outcome measures: cardiovascular and non-cardiovascular, 10- Publication status: Free access to the full record. The exclusion criteria included: 1- home-based CRP, 2- Participants with heart failure, heart transplants or implantable defibrillators, 3- records with pediatric or only old age participants. Search and Study Selection: The used electronic search strategy and selection process is based on the PRISMA flow diagram [35] as shown in (Fig. 1). Data Collection Process and Data Items: The data were extracted from the records' texts and/or in their tables and figures. Extracted data were presented in a table-based. The table included items of patients, intervention, comparison, outcome measure (PICO). Then, qualitative data analysis was done, and no quantitative statistical data analysis was applied. The COMs and NCOMs effects of exercise therapy interventions (AT, RT, and CT) were presented in another table. The review also included other resources in terms of the relevant articles within the reference list of the records included in the current SR. Data of these other resources are used to enrich the background, provide explanations, and emphasize the results of the analysis of the 10 included records. Assessment of Characteristics of Records: The Risk of bias assessment and assessment of study quality was conducted by quality scores determined by the PEDro Rating Scale (maximum score is 10 with the higher score reflecting greater methodological quality). This PEDro Rating Scale is designed to determine the adequacy of random allocation, concealed allocation, the similarity of groups at baseline, 


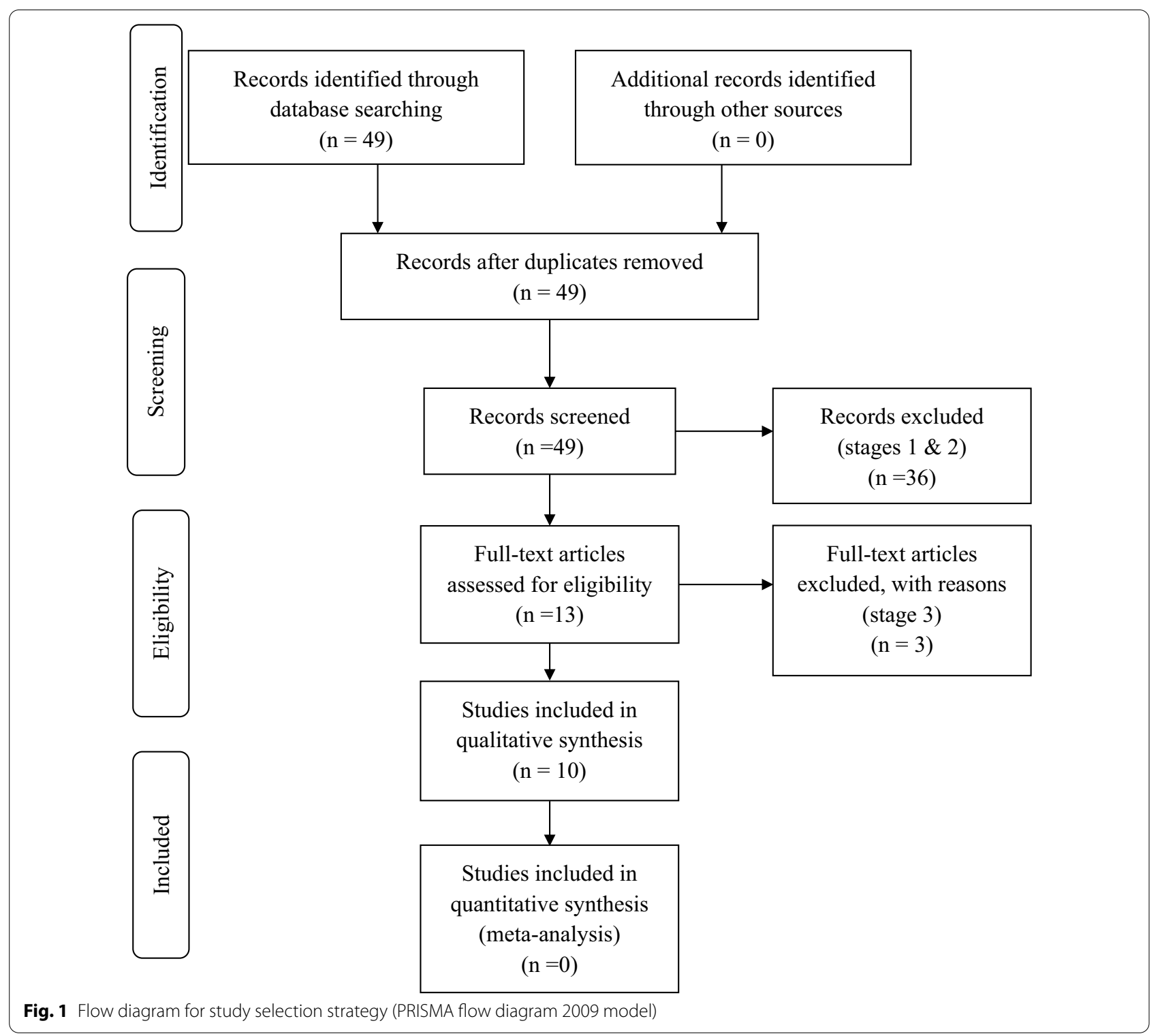

blinding of the subject, therapist, and/or assessor, measures of at least one key outcome from more than $85 \%$ of the subjects initially allocated to groups, intention-to-treat analysis, between-group analysis, and point estimates and variability.

The study selection and flow of records through the review resulted initially in 49 records. These 49 records were screened and assessed for eligibility at 3 stages as shown in the PRISMA flow diagram in Fig. 1. Out of these 49 records, 39 records were excluded based on the SR eligibility criteria. Reasons for exclusion are presented in Table 1. The studies that met the eligibility criteria and were included in the review were 10 (3 MA of RCTs and 7 RCTs). Other resources included 40 relevant articles which were in the reference list of the 10 studies.

The studies' characteristics and results of the included articles indicated that the most common inclusion criteria in the 10 studies included in the current SR were stable patients with ejection fraction $<45$ and with $\geq 50 \%$ or with $\geq 70 \%$ arterial diameter narrowing of at least one major coronary artery. Meanwhile, severe, or uncontrolled cardiac arrhythmia or unstable angina pectoris or uncontrolled hypertension, and musculoskeletal conditions limiting participation in exercise training were the most mentioned exclusion criteria within the 10 studies. Patients' age was of wide range in 4 studies (from $40^{\text {th }}$ to late $60^{\text {th }}$ or early $70^{\text {th }}$ ) and in one study it was of limited 
Table 1 Reasons of records exclusion through the 3 stages of study selection strategy

\begin{tabular}{|c|c|c|c|c|}
\hline $\begin{array}{l}\text { Stage of study } \\
\text { selection }\end{array}$ & & Reason of exclusion & & $\begin{array}{l}\text { Number of } \\
\text { excluded } \\
\text { records }\end{array}$ \\
\hline \multirow[t]{16}{*}{ Stage one } & Title relevancy & Irrelevant participants' diagnosis $(n=15)$ & Heart failure & 10 \\
\hline & & & Parkinson's disease & 1 \\
\hline & & & Spinal cord injury & 1 \\
\hline & & & Stroke & 1 \\
\hline & & & metabolic disorders & 1 \\
\hline & & & Atrial fibrillation & 1 \\
\hline & & Irrelevant participants'age $(n=3)$ & Old age only & 2 \\
\hline & & & Pediatric & 1 \\
\hline & & Irrelevant type of interventions $(n=4)$ & Exercise testing protocol & 1 \\
\hline & & & Weight loss protocol & 1 \\
\hline & & & Interval training & 1 \\
\hline & & & Inspiratory muscle training & 1 \\
\hline & & Irrelevant Language $(n=2)$ & French language & 1 \\
\hline & & & Icelandic language & 1 \\
\hline & Publication Date & Outdating (1989-2009) & & 10 \\
\hline & Total & & & 34 \\
\hline \multirow[t]{2}{*}{ Stage two } & Relevancy of Abstract & Irrelevant participants'age & Old age & 1 \\
\hline & & Irrelevant study design & Quasi-experimental design & 1 \\
\hline \multirow[t]{2}{*}{ Stage three } & Publication Status & Inaccessibility to full free articles & & 3 \\
\hline & Grand total & & & 39 \\
\hline
\end{tabular}

range (55-60 years) and in 3 studies the mean of patients' age was around 60 years. In all studies patients were of both gender except in 2 studies, only men were included. To study the effect of RT, different exercise-based protocols were used in the 10 studies. Four studies compared CT and AT. Two studies compared two CT protocols with different RT (RT of 2 sets X 12 repetitions Vs. RT of 3 sets X 15 repetitions in one study and concentric RT Vs. eccentric RT in the other study). In one study, a comparison of two CT protocols with different AT was found. Comparison between three protocols was also found in 2 studies: AT, RT, and CT in the first one and CT (RT of one set), CT (RT of 3 sets), and AT in the second study. The effect of isometric RT Vs. dynamic RT was found in one study. Duration of training protocol was varied; it lasts for about one month ( 4 or 5 weeks) (3 studies), for 2 months (one study) for 3 months (one study), for 6 months (one study), more than 7 months (one study), from 1 to 6 month (one study), from 1 to 7 months (one study) and from one to more than 7 months (one study). The range of frequency of RT protocol was from 2 times to 2-5 times/week. The intensity of the RT was determined through one of the following measures; a percentage from the one-repetition maximum (1 RM) (6 studies) (range from $30 \%$ to over $80 \%$ of the $1 \mathrm{RM}$ ), a percentage from the maximum voluntary contraction ( 2 studies) in one study $70 \%$ of isometric maximum voluntary contraction and in another study $60 \%$ of dynamic maximum voluntary contraction, heart rate reserve (HRR) (one study) (75-85\% of HRR), ratings of perceived exertion of Borg scale ( 2 studies) with the weight to elicit a score of 11-15 in one study and weight to elicit a score of 4-6 on a modified Borg scale of 1 through 7 in the other study. Regarding the volume of RT, the range of the number of sets was from one to 8, the range of exercise repetitions was from 10 to 20 and the number of exercises ranged from 2 to 10 exercises. The AT protocol was also of variant duration (ranged from 8 to $60 \mathrm{~min}$ ) and variant intensity (ranged from 60 to $80 \%$ of peak oxygen consumption $\left(\mathrm{VO}_{2}\right.$ peak) in one study, $40-85 \%$ of peak heart rate in one study and $60 \%-70 \%$ of HRR in another study). The 10 studies included cardiovascular outcome measures (COMs) and non-cardiovascular outcome measures (NCOMs). Within the COMs were the exercise capacity in terms of $\mathrm{VO}_{2}$ peak or $\mathrm{VO}_{2}$ max (4 studies), maximum metabolic equivalent (MET) (one study), exercise time during symptom-limited cardiopulmonary exercise testing (one study), the left ventricle ejection fraction (LVEF) ( 2 studies) and cardiac output (one study). Among the NCOMs were SM strength (6 studies) and SM endurance (one study). The SM strength included lower extremity, upper extremity, and trunk muscles. The HRQOL 
was also a common NCOM as it was used in 5 studies. Other NCOMs included the body composition (2 studies) and body weight and body mass index (BMI) (one study). Adverse effects or side effects were measured in 4 studies. Summary of the basic characteristics of the 10 included studies is presented in Table 2 in terms of PICO in addition to the grade quality of evidence in a score out of 10. The summary data for all effects of exercise therapy including the AT, RT, and CT are shown in Table 3. The presented effects of exercise therapy included COMs and NCOMs.

The results of the effects of resistant training of this SR revealed that adding RT to the AT in CT exercise-based protocol resulted in favorable improvement in COMs and NCOMs for adult CAD patients. In 4 studies (2 MA of RCTs and 2 RCTs), out of the 10 studies included in this SR, there was a significant improvement for CT more than the AT alone in different COMs and NCOMs as exercise capacity $\left(\mathrm{VO}_{2}\right.$ peak, exercise time), $\mathrm{HRV}$, upper and lower extremities SM strength and leg press muscle endurance, body composition and HRQOL [2, 10, 37-39]. In the RCT [39] in which 2 CT (eccentric RT Vs. concentric RT) were compared, results showed that there was a tendency for a better improvement symptom-limited $\mathrm{VO}_{2}$ and ankle plantar flexor isometric maximum voluntary contraction in the eccentric group. Meanwhile, the RCT that compared between 2 different RT protocols (isometric Vs. dynamic), indicated that there was a pre-post significant increase in peak power output with favorable to isometric protocol [40]. The pre-post training significant increase in maximal HR and systolic blood pressure (SBP) was with no significant difference between the isometric and dynamic protocols. The isometric RT protocol significantly decreased the maximal diastolic blood pressure (DBP) while the dynamic protocol significantly increased it. The upper and lower extremities SM strength was significantly increased with both protocols with no significant difference. Both protocols had no effect on the body composition nor in the anthropometric variables (BMI), waist circumference). In the second RCT [34] that compared between 2 CT (RT of 2 sets X 12 repetitions Vs. 3 sets $\mathrm{X} 15$ repetitions), both RT volumes produced pre-post improvement in CVOMs $\left(\mathrm{VO}_{2}\right.$ peak) and NCOMs (SM strength) with no significant difference between both volumes. It was remarkable that although AT alone did not change lipid profiles (high-density lipoprotein) or HRQOL (Self-evaluated health), the addition of RT did [33]. Results proved that RT is safe for stable CAD patients as no side effects or adverse effects were observed [34, 39, 40].

To discuss the results of this SR, it can be claimed that the AT is the gold standard protocol in CRP for CAD patients and in the past 3 decades, RT started to be added to the AT. The decrease of exercise capacity of patients with CAD causes limitation in their physical activity, musculoskeletal fitness, and functional capacity which markedly affect their HRQOL, especially the physical component. This reason raised the need for another protocol, in addition to the AT, to improve their functional capacity. The RT was the perfect candidate for this purpose as it is well known to improve musculoskeletal fitness in terms of SM strength as well as endurance. Results of this SR can provide the physical therapy clinicians with the clear consensus that RT is an effective and safe exercise therapy as added to AT in exercise-based CRP for patients with CAD. The positive effects of RT were reported for both the COMs and NCOMs.

The addition of RT to AT resulted in increased improvement of exercise capacity, $\mathrm{VO}_{2}$ peak [2,37], and this increase may be related to the improvement in the SM strength associated with RT which enabled patients to participate more effectively in the CRP and benefit more from the cardiovascular health improvement associated with AT. It was proved that SM strength of CAD patients is lower than that of age-matched subjects [41] and impaired SM strength is strongly associated with poor exercise capacity [42], so it could be hypothesized that improvement in SM strength would improve the exercise capacity. This hypothesis is supported by the fact that AT may be discontinued because of muscular pain because of muscle fatigue due to SM weakness.

The RT was claimed to elevate the BP, particularly SBP, which could be risky for CAD patients, and this claim delayed RT joining to CRP up to the early $1990^{\text {th }}$. The current SR acts as proof to discontinue this claim for 3 reasons. 1- there was no observed side effects or adverse events in $\mathrm{CT}$ with added RT which indicates that the RT is as safe as AT. 2- the isometric RT reduced the DBP in one study [40] of the review and it produced no change in both SBP and DBP in another study [33]. It was suggested that the undesirable elevations in SBP and HR occur when the duration of RT exceeds around 10 repetitions per set, [43] so it is applicable that physical therapy clinicians can design the RT in sets of 10 repetitions with an interval resting of $30 \mathrm{~s}$ between sets. 3- low-intensity RT with 15 to 20 repetitions resulted in only a modest elevation in BP, like the hemodynamic changes observed with AT $[16,44]$, and this intensity and repetitions could be considered in describing RT. The current SR even indicates the possible anti-hypertensive effect of the RT as in one study of the review the systolic and diastolic aortic pressure were measured as indicators for the central $\mathrm{BP}$ and RT had a similar result in decreasing the systolic pressure as the AT and even RT decreased the diastolic pressure while the AT produced no change post-training [1]. Different mechanisms could be responsible for 
Table 2 The basic characteristics of articles included in the systematic review

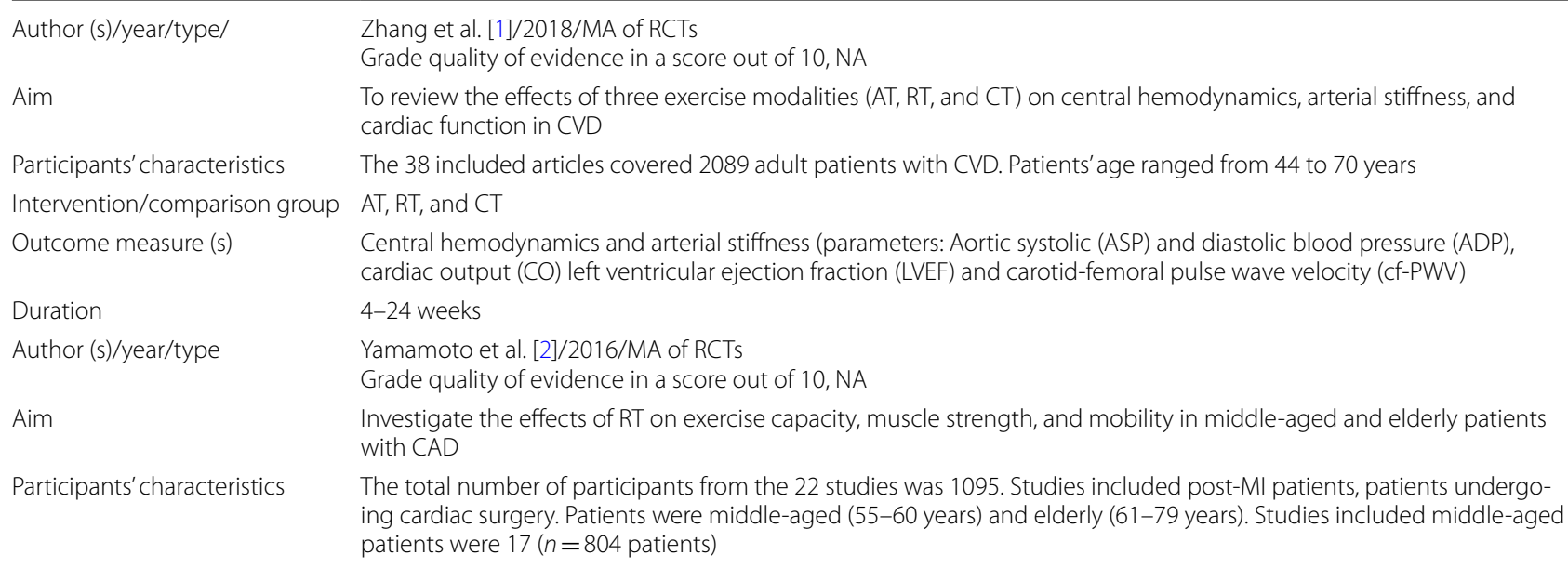

Intervention/comparison group Exercise group: RT or CT. The intensity of RT ranged from 50 to $69 \%$ of 1 repetition maximum (1RM) (1RM: heaviest weight lifted once). Mode of exercise: weight training Control group: Usual care or AT. The meta-analysis compared the RT with the usual care or the CT (AT + RT) with the AT alone

Outcome measure (s) Lower extremity muscle strength (Isometric and isokinetic knee extension torque), upper extremity muscle strength (the 1-RM of chest press, or bicep curl), exercise capacity (Peak oxygen consumption (peak $\mathrm{VO}_{2}$ ) or exercise time (the sum of exercise time excluding rest and warm-up periods) during symptom-limited cardiopulmonary exercise testing (CPX), mobility (household physical activity and functional mobility scores from continuous-scale physical performance tests)

Duration Training duration in the middle-aged group ranged from 4-8 weeks. 2-5 times/week

Author (s)/year/type Marzolini et al. [37]/2011/MA of RCTs Grade quality of evidence in a score out of 10, NA

Aim To examine effects of combined training (CT) of RT + AT versus AT alone in CAD

Participants' characteristics Out of the 12 studies included, 229 patients received AT and 275 patients received CT. Age ranged from 49 to 71 years Intervention/comparison group

Outcome measure (s) The CT groups prescribed 8-60 min of AT, 2-4 times/week at $40-85 \%$ of pea
sisted of 2-4 sets, of 2-10 exercises at an intensity of $40-80 \%$ of the 1 RM

Duration Body composition, exercise capacity $\left(\mathrm{VO}_{2}\right.$ peak), strength, and health-related quality-of-life (HRQOL)

Author (s)/year/type 4-29 weeks, 2-3 times/week Aim $\checkmark$ Gremeaux Pole et al. [39]/2010/RCT

Participants' characteristics To compare the effects between concentric and eccentric RT on exercise capacities in patients with CAD Intervention/comparison group Fourteen patients aged between 40 and 65 years with stable CAD after percutaneous coronary intervention Patients performed two 30-min sessions of two different AT (bicycle and arm cycling). Patients then performed 3 sets of 12 contractions in $30 \mathrm{~s}$ for each group, followed by a 30-s rest period, with a load corresponding to 50\% of 1RM. In the concentric group, bicycle work was performed three times a week, on a standard cycle ergometer In the eccentric group subjects also trained three times a week, but on a specifically constructed cycle ergometer, with a motor driving the pedals in the reverse direction

Outcome measure (s) Symptom-limited $\mathrm{VO}_{2}$, peak workload, isometric strength of leg extensor and ankle plantar flexors, distance covered during the 6-min walk test, and time to perform the 200-m fast walk test

Duration 5 weeks, 3 times/week

Author (s)/year/type

Dor-Haim et al. [10]/2018/RCT

Grade quality of evidence in a score out of 10 , is 6

Aim

To compare the effectiveness of continuous AT (CAT) to super-circuit training (SCT), (SCT = combined RT and AT in interval)

$\begin{array}{ll}\text { Participants' characteristics } & 29 \text { men post-MI participants aged from 47-69 years, ejection fraction < 45\%), stable and were able to attend regularly } \\ \text { supervised exercise program, New York Heart Association Classification I-III }\end{array}$ 
Table 2 (continued)

Intervention/comparison group CAT patients $(n=15)$ exercised at 60\%-70\% of their heart rate reserve and SCT patients $(n=14)$ exercised at $75-85 \%$ of their heart rate reserve.

Each CAT session lasts for 45 min. Each SCT set included one RT set, 3 min of aerobic interval, and a resting period. This sequence was repeated eight times ( 8 sets). Exercise intensity was determined using the heart rate reserved method (i.e., maximal heart rate-resting heart rate) The patient's maximal heart rate was established via a baseline graded exercise tolerance test.

The RT was composed of 8 different exercises, namely, horizontal rowing, chest press, leg press, shoulder press, leg extension, lateral pull-down, leg flexion and assisted squat. Each exercise consisted of one set of 15 repetitions. In the first two weeks of the program, the training intensity was light (30\% of 1RM) and progressively increased to $50 \%$ of 1RM

Outcome measure (s) Cardiac function (LVEF, and aerobic functional capacity Aerobic fitness), Handgrip strength, and HRQOL (Medical Outcomes Study Short-Form Health Survey (SF-12).

To evaluate the feasibility and safety of the SCT, data regarding the program's major and minor adverse events (e.g., hospitalization, syncope, arrhythmia, muscle ache) were recorded. In addition, information regarding reasons for attrition from the program was evaluated

Duration

12-weeks, 2 times/week

Author (s)/year/type

Guiraud et al. [40]/2017/RCT

Grade quality of evidence in a score out of 10 , is 6

Aim

To compare 2 different modes of RT, an isometric mode with the Huber Motion Lab (HML) and traditional strength training (TST) dynamic muscle contraction, in CAD patients undergoing a CR program

Participants' characteristics

HML group $(n=25)$ and TST group $(n=25)$. Out of the 50 patients, $94 \%$ were men.

Inclusion criteria: patients with $\geq 70 \%$ arterial diameter narrowing of at least one major coronary artery and/or documented previous MI.

Exclusion criteria: patient with the recent acute coronary syndrome ( $\leq 1$ month), significant resting electrocardiography abnormality, severe arrhythmia, history of congestive heart failure, uncontrolled hypertension, bypass surgery $\leq 3$ months, percutaneous coronary intervention $\leq 1$ month, LVEF $\leq 45 \%$, pacemaker installation, modification of medication $<2$ weeks, and musculoskeletal conditions making exercise on a cycle ergometer difficult or contraindicated

Intervention/comparison group The HML is a motorized rotating platform that allows patients to perform exercises that simultaneously involve balance, coordination, and strength training.

HML sessions (isometric): 6 exercise blocks in different postures. Each block consisted of 8 contractions of $6 \mathrm{~s}$ alternating with 10 s of passive recovery, repeated twice. The total duration of the session, which included a 3-min warm-up, 10 min of maximum voluntary contraction (MVC) assessment, 27 min of exercise, and 5-min recovery, was 45 min. The intensity of isometric contraction was set at 70\% of the MVC. Because the MVC was calculated at each session, the exercise intensity was automatically adapted to enable progression.

TST sessions (dynamic) involved circuit training including 6 different machines: leg press, chest press, vertical traction (shoulder press), low row (working back), pectoral (butterfly), and leg extension. Movements allowed for dynamically working for the same muscle groups as with the HML. The intensity was set at $60 \%$ of MVC. At each position, patients were asked to repeat 3 series of 12 repetitions. With TST, the MVC was calculated by using the one-repetition maximum test (1-RM) on each machine at the beginning of each week during the program

Outcome measure (s)

Cardiopulmonary exercise test (The peak power output (PPO), maximal HR, maximal SBP, maximal DBP), maximal upper and lower limbs isometric voluntary contraction, endothelial function (Reactive hyperemia index (RHI) and Anthropometric variables (BMI, waist circumference), body composition (bioelectrical impedance), HRQOL (French version of the Medical Outcomes Study Short Form 36 (SF-36) and sleep quality (The Pittsburgh Sleep Quality Index (PSQI)

Duration

4 weeks, 4 times/week

Author (s)/year/type

Caruso [38]/2015/RCT

Grade quality of evidence in score out of 10, is 6

Aim

To investigate the effects of high repetition/low load RT (HR/LL-RT) program on heart rate variability (HRV) and muscular strength and endurance in CAD patients

Participants' characteristics

Twenty male patients with CAD were randomized to control the usual AT care group (UCG) (61 \pm 4.4 years) or CT (RTG) with RT (HR/LL-RT) and AT (61.3 \pm 5.2 years).

Inclusion criteria consisted of 1) an established clinical diagnosis of CAD for at least one year; 2) New York Heart Association (NYHA) classification

I-II; 3) medical management for at least one year according to the American Heart Association/American College of Cardiology recommendations; and

4) participation in a CR program for at least 1 year, exclusively comprised of AT.

Exclusion criteria consisted of 1) the presence of uncontrolled cardiac arrhythmias; 2) unstable angina pectoris, uncontrolled hypertension, or

pulmonary and renal comorbidities; 3) conditions limiting participation in exercise training (i.e., orthopedic limitations and musculoskeletal disorder): and 4) abnormal hemodynamic responses during a previous incremental exercise testing 
Table 2 (continued)

Intervention/comparison group The UCG program consisted of $1 \mathrm{~h}$ of AT, divided into 10 min of warm-up (stretching of upper and lower limbs), 20-30 min of treadmill or cycle ergometer training at an intensity of 70\% of maximal HR obtained by exercise testing, cool-down (low-intensity dynamic exercises and stretching upper and lower limbs) and a relaxation phase (10 min). The AT program was performed 2 times per week and continued in all patients in both groups (UCG and RTG). Formal clinic evaluations by a cardiologist occurred every 6 months and exercise testing on a cycle ergometer was performed at 3-month intervals to adjust the intensity of the AT program.

Patients that were allocated to the RTG were instructed to continue the CR program and in parallel perform the RT protocol (2 times/week for 8 weeks on days the CR program was not performed). Each session lasted $1 \mathrm{~h}$ and consisted of an arm-up, HR/LL-RT on a leg press, and a cool-down period.

The warm-up and cool-down session consisted of lower limb stretching. Subjects underwent 3 sets over 2 min at a movement rhythm of 10 repetitions per minute, maintaining respiratory cadence (as the volunteer had been oriented during the 1-RM test). Each repetition was performed in $5 \mathrm{~s}$ ( $2 \mathrm{~s}$ of extension and $3 \mathrm{~s}$ of knee and hip flexion), with the rhythm controlled by verbal commands. Each set was separated by 5 min rest intervals. The intensity of exercise training was 30\% of 1-RM, a resistance approximating the lactate threshold (LT) in a previous investigation.

RTG received HR/LL-RT program of 45 degrees leg press 3 sets of 20 repetitions. The initial load was set on $30 \%$ of $1-\mathrm{RM}$

Outcome measure (s) Resting HRV

Muscle strength (1 repetition maximum (1-RM) exercise test on leg press)

Muscle endurance

Duration

8 weeks, 2 times/week

Author (s)/year/type

Berent et al. [34]/2011/RCT

Grade quality of evidence in a score out of 10 , is 6

Aim

To compare the effectiveness of 2 different volumes of RT combined with AT in residential CR

Participants' characteristics

Patients ( $N=295$ ) (71 women, 224 men) with a mean age of 62.7 years.

Included in the study were patients after a cardiovascular event with and without percutaneous coronary intervention and stent implantation or after heart surgery. Exclusion criteria were a pericardial effusion with a hemodynamic effect, pleural effusions responsible for dyspnea, acute infectious diseases, wound infections after surgery, and unstable angina

Intervention/comparison group Group I (134 patients): 2 sets X 12 repetitions (REPS) and Group II (161 patients): 3 sets X 15 REPS per session, 2 times per week; each RT session consisting of 10 different resistance exercises (back extension, back flexion, reversed chest butterfly, chest butterfly, leg press, rowing machine, leg extension, sitting leg curl, pull down, and dips). In addition, patients also completed continuous moderate intensity AT composed of cycle ergometry 6 times per week for $17 \pm 4$ min (mean \pm SD) and walking 5 times per week for 45 min.

The intensity of the RT was individualized for each patient. Patients performed RT at the ratings of perceived exertion (RPE) between 4 and 6 on a modified Borg scale of 1 through 7 (which was considered moderate intensity), and the desired RPE was determined when patients were lifting weights between 13 and 15 REPS of a given RT exercise. Stress perception for RT was maintained at an RPE of 4-6 for each training session followed by an increase in weight/ load as patients improved in RT during residency. Avoiding determination of 1RM, the load-repetition relationship for RT was approximated and resulted in $50 \%$ to $60 \%$ of the 1 RM

Outcome measure (s) Exercise capacity $\left(\mathrm{VO}_{2} \mathrm{max}\right), \mathrm{HR}$, SBP, DBP, muscle strength, blood biochemistries (lipids), body weight and body mass index (BMI)

Duration

About 4 weeks ( $26 \pm 4$ (mean \pm SD) days) the RT is applied 2 times/week

Author (s)/year/type

Marzolini et al. [52]/2015/RCT

Grade quality of evidence in a score out of 10 , is 4

Aim

To compare the effects of AT combined with RT (1 versus 3 sets) versus AT alone on HRQOL and psychosocial outcomes

Participants' characteristics patients with CAD participating in Outpatient Cardiac Rehabilitation Program. Fifty-three patients (mean \pm SD age $60.6 \pm 10.6$ years) completed training.

Patients have $\geq 50 \%$ block in at least one major coronary artery. They were post-percutaneous procedures or CABG

Intervention/comparison group All patients received AT ( 5 days/week) for the first 5 weeks then they were divided into three groups during the rest of the 24 weeks of the training duration (total 29 weeks).

Group (1) ( $n=16)$ continued to receive the AT alone (5 days/week),

Group (2) $(n=19)$ received AT (3 days/week) + RT (one set, 2 days/week), Group (3) $(n=18)$ received AT (3 days/ week) + RT (3 sets, 2 days/week).

The AT included $30 \mathrm{~min}$ of walking and/or jogging $\left(1.6 \mathrm{~km}\right.$ and $\left.60 \% \mathrm{VO}_{2 \text { peak. }}\right)$. The prescription of AT was progressed every 2 weeks with a maximum of $60 \mathrm{~min}, 6.4 \mathrm{~km}, 80 \% \mathrm{VO}_{2}$ peak.

The RT started with $60 \%$ of 1 RM and progressed to $70-75 \% 1$ RM and the repetition progressed from 10 to 15 repetitions. Upper body exercise included 5 exercises +2 exercises to the trunk. The lower body exercises included 3 exercises

Outcome measure (s) HRQOL and psychosocial parameters were assessed before and after 29 weeks of training by questionnaire (SF-36 and depression score). self-efficacy of lower and upper body physical activity, depression, and physical component of HRQOL 
Table 2 (continued)

\begin{tabular}{|c|c|}
\hline Author (s)/year/type & $\begin{array}{l}\text { Currie et al. [33]/2015/RCT } \\
\text { Grade quality of evidence in a score out of } 10 \text {, is } 4\end{array}$ \\
\hline Aim & $\begin{array}{l}\text { 1. To compare between } 2 \text { protocols of AT; the low-volume high-intensity interval training (Low volume HIIT) and the } \\
\text { moderate-intensity continuous training (MICT). } \\
\text { 2. To determine the effect of combining RT to the above-mentioned } 2 \text { AT on cardiovascular risk profiles in patients } \\
\text { with CAD }\end{array}$ \\
\hline Participants' characteristics & $\begin{array}{l}\text { Nineteen patients ( } 2 \text { females) with CAD recruited during phase II cardiac rehabilitation outpatient program. Inclusion } \\
\text { criteria included a recent ( }<3 \text { months) CAD event, which was defined as the patient having at least one of the fol- } \\
\text { lowing: myocardial infarction, percutaneous coronary intervention, or coronary artery bypass graft; angiographically } \\
\text { documented stenosis } \geq 50 \% \text { in at least one major coronary artery; positive exercise stress test determined by symp- } \\
\text { toms of chest discomfort accompanied by electrocardiographic (ECG) changes of }>1 \mathrm{~mm} \text { horizontal or down sloping } \\
\text { ST-segment depression, or a positive nuclear scan }\end{array}$ \\
\hline Intervention/comparison group & 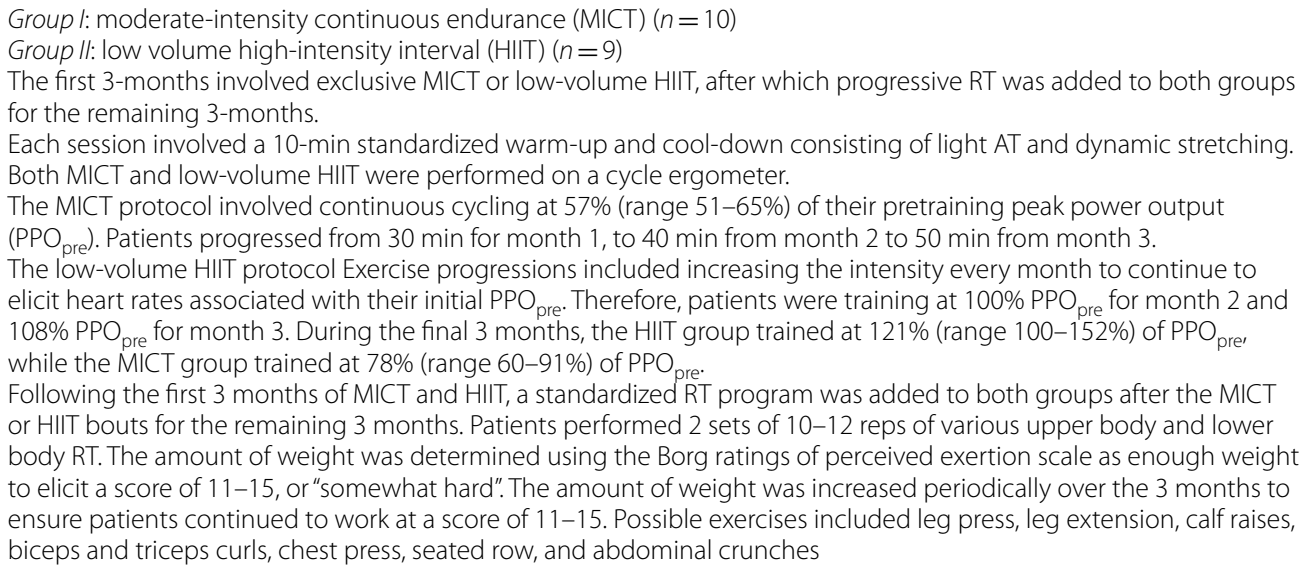 \\
\hline Outcome measure $(\mathrm{s})$ & $\begin{array}{l}\text { Exercise capacity }\left(\mathrm{VO}_{2} \text { peak), blood pressure and heart rate, lipid profiles and } \mathrm{HRQOL} \text { assessments were performed at }\right. \\
\text { pretraining, } 3 \text { and 6-months training }\end{array}$ \\
\hline Duration & 24 weeks, 2 times/week \\
\hline
\end{tabular}

the reduction of central BP with RT including improved microvascular perfusion, peripheral muscular artery dilation, and peripheral vascular resistance. And in another study of the review, the RT as part of CT produced no change in both SBP and DBP [33].

The current SR identified that CT with added RT had an advantage to improving upper and lower extremities SM strength and endurance compared to AT alone. This improvement was confirmed in other studies [21, 22, 45, 46] and it is important from the HRQOL point-of-view as it allows individuals to perform activities of daily living and work-related tasks. It also positively affects proprioceptive abilities, thereby leading to a gradual improvement in coordination and gait control which reduces the risk of falls thereby maintaining functional independence for a greater period especially in elderly CAD patients $[47,48]$. In addition, it reduces cardiac demands (HR and SBP) when lifting a given load [49].

Adding RT to AT produced more improvement in body composition than AT alone. The proposed reasons for this improvement are that RT promotes greater muscle mass than AT, and thus may contribute to fat mass loss via the increase in the resting metabolic rate $[37,50]$ and that RT increased SM endurance allowing subjects to perform activities with long duration and result in more burn of fat as a source of fuel. This improvement has clinical implications because there is a growing prevalence of obesity among CAD patients and as the amount of fat mass increases especially abdominal fat, it elevates blood lipid levels, BP, and risk of CAD [37]. That is why weight loss, particularly trunk fat loss, became CRP basic goal and RT is the key protocol to address this issue [37, 51].

The current SR adopts the hypothesis that improvement in the exercise capacity and SM strength and endurance are logically to be reflected functionally and improve the CAD patients' HRQOL. The adopted hypothesis is in line with the opinion that improvement in SM strength benefits to reduce risk of injury from falls, improved ability to perform activities of daily living and help to promote independent living, which are key components of HRQOL [48]. Also, when the CAD patients get rid of the limitation of exercise capacity which restricts their functional abilities, and become physically free and able to perform the daily activities independently, the mental status would improve in the forms of decreased depression level and the improved 


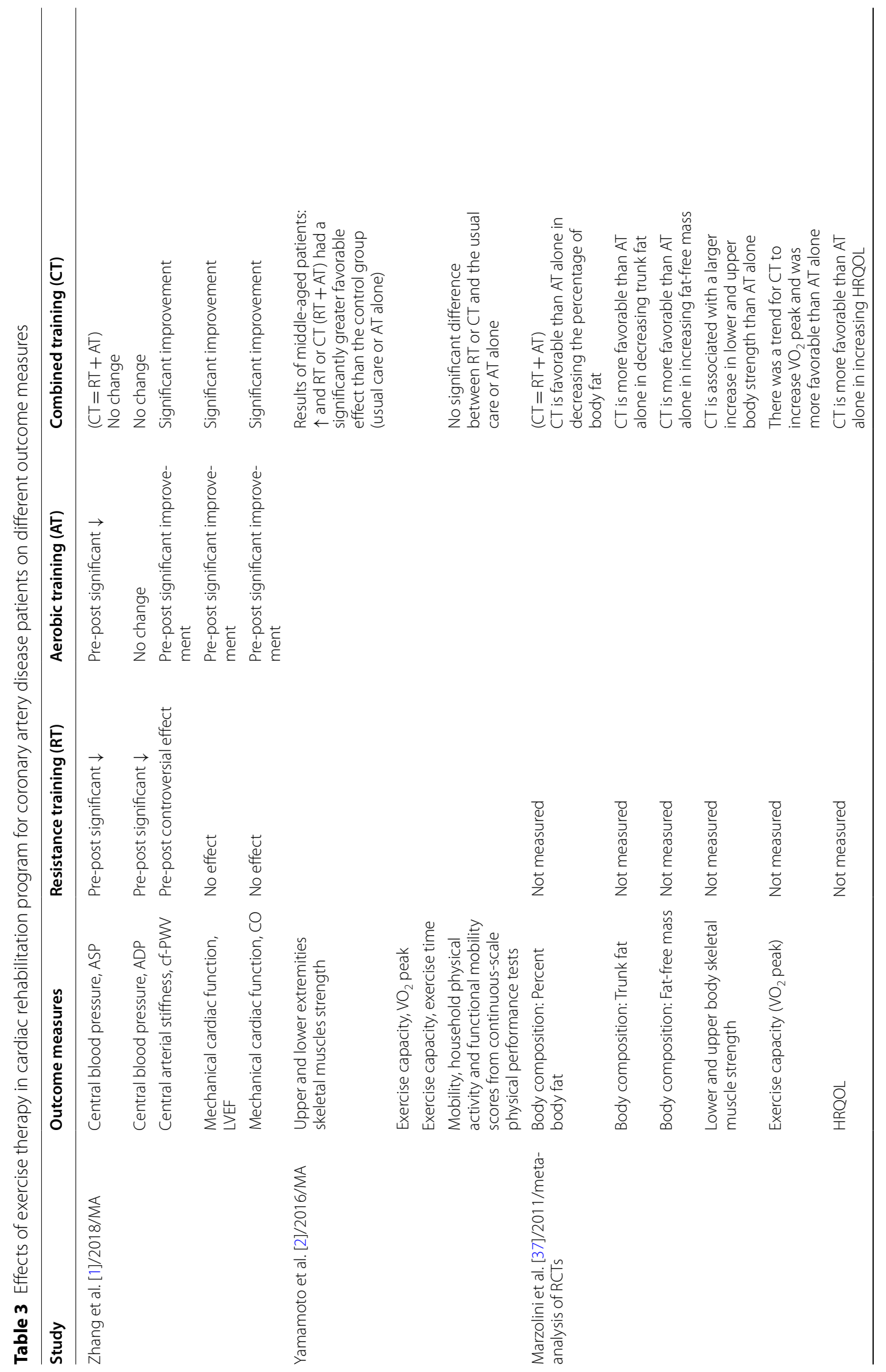



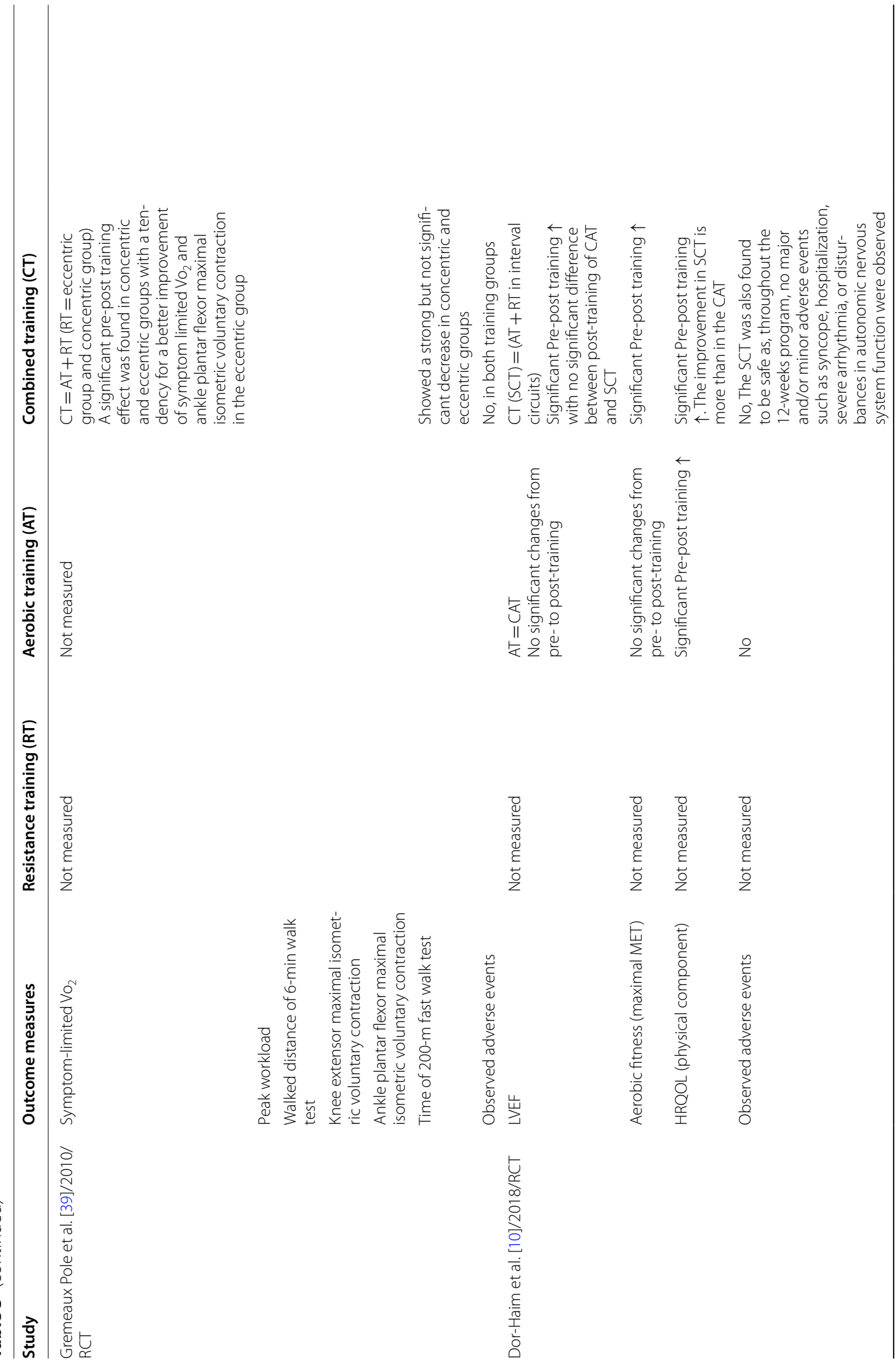

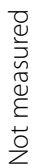
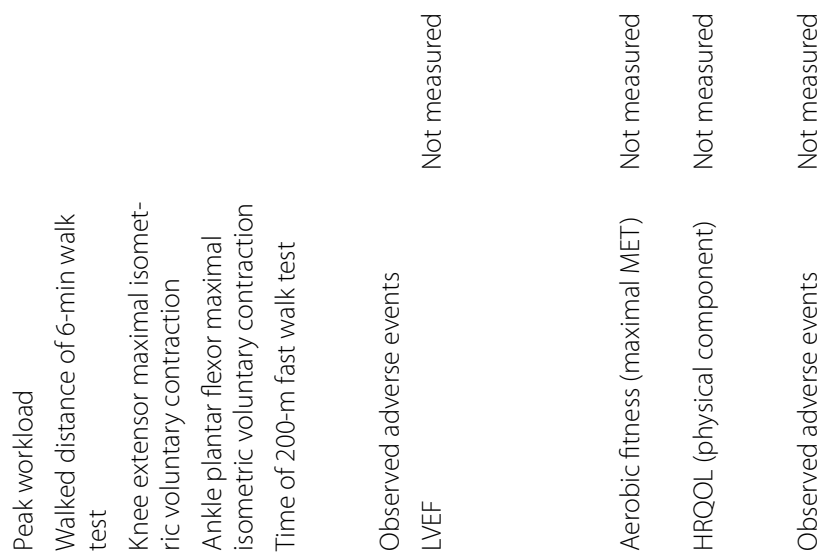

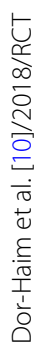




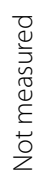
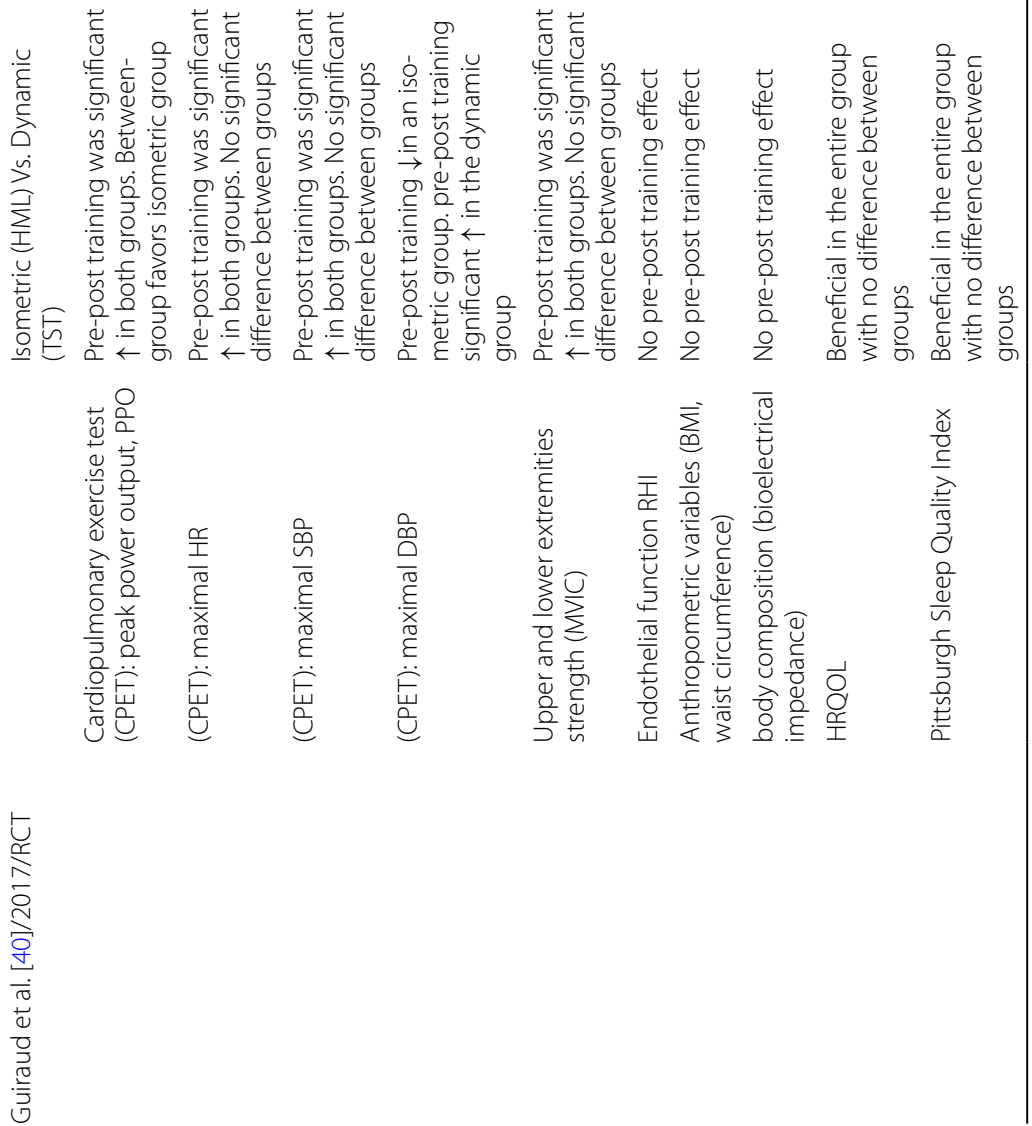


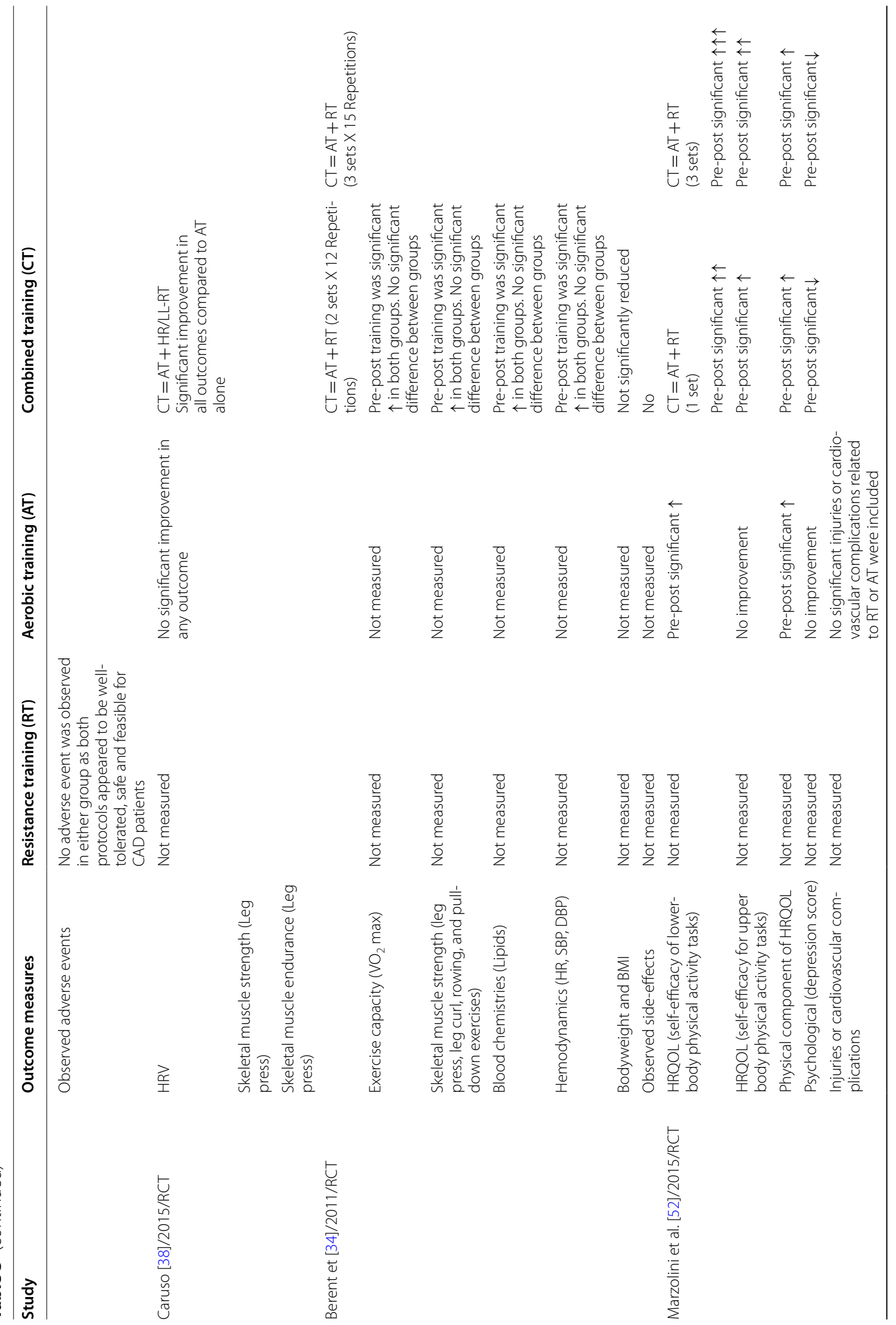




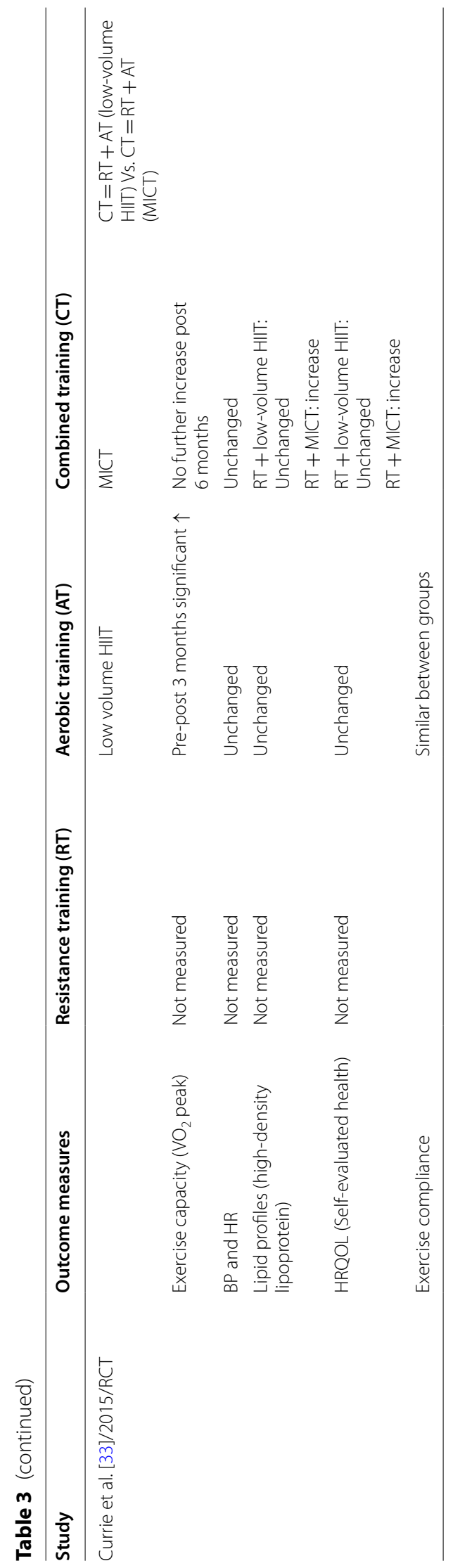


quality of sleep. It was interesting to find out that the AT alone could not improve the HRQOL, and the recorded improvement was related to CT with RT addition [52].

\section{Conclusions}

The RT should be considered as an integral exercise therapy of exercise-based CRP for stable CAD patients. It is safe as well as the AT. The CT with added RT to AT has beneficial effects on COMs and NCOMs even more than the AT alone. The effectiveness of RT includes improvement in exercise capacity $\left(\mathrm{VO}_{2}\right.$ peak and exercise time), $\mathrm{BP}$, SM strength and endurance, body composition, sleep quality, depression, and HRQOL.

This SR had implications for physical therapy clinical practice as it highlighted the following clinical issues for exercise-based CR program for CAD adult patients; 1 - stopping contra-indicating the RT for the false claim that RT produces undesirable elevated BP and even start thinking about its anti-hypertensive effect, 2- inclusion of losing bodyweight and improving body composition within the goals, 3- eccentric and isometric RT would be the preferred RT protocol.

Limitations of the study include that only one author reviewed the records. Although the PEDro is the best and the most trustable evidence-based electronic database and search engine, it was the only electronic search engine used.

\begin{abstract}
Abbreviations
CRP: Cardiac rehabilitation program; CAD: Coronary artery disease; SR: Systemic review; RCTs: Randomized controlled trials; MA: Meta-analysis; PRISMA: The Preferred Reporting Items for Systematic Reviews and Meta-Analyses; CABG: Coronary artery bypass graft; RT: Resistant training; AT: Aerobic training; $\mathrm{CT}$ : Combined training; HRQOL: Health-related quality of life; COMs: Cardiovascular outcome measures; NCOMs: Non-cardiovascular outcome measures; $\mathrm{VO}_{2}$ : Oxygen consumption; HR: Heart rate; HRR: Heart rate reserve; HRV: Heart rate variability; SBP: Systolic blood pressure; DBP: Diastolic blood pressure; SM: Skeletal muscles; BMI: Body mass index; MET: Metabolic equivalent; LVEF: Left ventricle ejection fraction.
\end{abstract}

\section{Acknowledgements}

Not applicable.

\section{Authors' contributions}

The author declares that she is a single only author for this article. The author read and approved the final manuscript.

\section{Funding}

The author declares that there is no received funding.

\section{Availability of data and materials}

The datasets analyzed during the current systematic review study are available on the PEDro web page at https://pedro.org.au/.

\section{Declarations}

Ethics approval and consent to participate Not applicable.
Consent for publication

Not applicable.

\section{Competing interests}

The author declares that there are no competing interests.

Received: 2 June 2021 Accepted: 1 February 2022

Published online: 10 February 2022

\section{References}

1. Zhang Y, Qi L, Xu L, Sun X, Liu W, Zhou S, Van de Vosse F, Greenwal SE (2018) Effects of exercise modalities on central hemodynamics, arterial stiffness and cardiac function in cardiovascular disease: systematic review and meta-analysis of randomized controlled trials. PLOS ONE 13(7):e0200829. https://doi.org/10.1371/journal.pone.0200829

2. Yamamoto S, Hotta K, Ota E, Mori R, Matsunaga A (2016) Effects of resistance training on muscle strength, exercise capacity, and mobility in middle-aged and elderly patients with coronary artery disease: a metaanalysis. J Cardiol 68:125-134

3. Balady GJ, Williams MA, Ades PA, Bittner V, Comoss P, Foody JM, Franklin B, Sanderson B, Southard D, American Heart Association Exercise, Cardiac Rehabilitation, and Prevention Committee, the Council on Clinical Cardiology; American Heart Association Council on Cardiovascular Nursing; American Heart Association Council on Epidemiology and Prevention; American Heart Association Council on Nutrition, Physical Activity, and Metabolism; American Association of Cardiovascular and Pulmonary Rehabilitation (2007) Core components of cardiac rehabilitation/secondary prevention programs: 2007 update: a scientific statement from the American Heart Association Exercise, Cardiac Rehabilitation, and Prevention Committee, the Council on Clinical Cardiology; the Councils on Cardiovascular Nursing, Epidemiology and Prevention, and Nutrition, Physical Activity, and Metabolism; and the American Association of Cardiovascular and Pulmonary Rehabilitation. Circulation 115(20):2675-2682

4. Pedersen BK, Saltin B (2006) Evidence for prescribing exercise as therapy in chronic disease. Scand J Med Sci Sports 16(suppl 1):3-63

5. Antman EM, Anbe ST, Armstrong PW, Bates ER, Green LA, Hand M, Hochman JS, Krumholz HM, Kushner FG, Lamas GA, Mullany CJ, Ornato JP, Pearle DL, Sloan MA, Smith SC Jr, American College of Cardiology; American Heart Association; Canadian Cardiovascular Society (2004) ACC/AHA guidelines for the management of patients with ST-elevation myocardial infarction: executive summary: a report of the American College of Cardiology/American Heart Association Task Force on Practice Guidelines (Writing Committee to Revise the 1999 Guidelines for the Management of Patients With Acute Myocardial Infarction) [published correction appears in J Am Coll Cardiol. 2005; 45:1376]. J Am Coll Cardiol 44:671-719

6. Braunwald E, Antman EM, Beasley JW, Califf RM, Cheitlin MD, Hochman JS, Jones RH, Kereiakes D, Kupersmith J, Levin TN, Pepine CJ, Schaeffer JW, Smith EE III, Steward DE, Theroux P, Gibbons RJ, Alpert JS, Faxon DP, Fuster V, Gregoratos G, Hiratzka LF, Jacobs AK, Smith SC Jr (2002) ACC/AHA 2002 guideline update for the management of patients with unstable angina and non-ST-segment elevation myocardial infarction: summary article: a report of the American College of Cardiology/American Heart Association Task Force on Practice Guidelines (Committee on the Management of Patients With Unstable Angina). J Am Coll Cardiol 40:1366-1374

7. Gibbons RJ, Abrams J, Chatterjee K, Daley J, Deedwania PC, Douglas JS, Ferguson TB Jr, Fihn SD, Fraker TD Jr, Gardin JM, O'Rourke RA, Pasternak RC, Williams SV, Alpert JS, Antman EM, Hiratzka LF, Fuster V, Faxon DP, Gregoratos G, Jacobs AK, Smith SC Jr (2003) ACC/AHA 2002 guideline update for the management of patients with chronic stable angina: summary article: a report of the American College of Cardiology/American Heart Association Task Force on Practice Guidelines (Committee on the Management of Patients With Chronic Stable Angina). Circulation 107:149-158

8. Global Atlas on Cardiovascular Disease Prevention and Control. Geneva: World Health Organization, 2011. file:///F:/9789241564373_eng.pdf

9. Lloyd-Jones D, Adams RJ, Brown TM, Carnethon M, Dai S, De Simone G, Ferguson TB, Ford E, Furie K, Gillespie C, Go A, Greenlund K, Haase N, Hailpern S, Ho PM, Howard V, Kissela B, Kittner S, Lackland D, Lisabeth L, 
Marelli A, McDermott MM, Meigs J, Mozaffarian D, Mussolino M, Nichol G, Roger VL, Rosamond W, Sacco R, Sorlie P, Stafford R, Thom T, WasserthielSmoller S, Wong ND, Wylie-Rosett J, American Heart Association Statistics Committee and Stroke Statistics Subcommittee (2010) Executive summary: heart disease and stroke statistics-2010 update: a report from the American Heart Association. Circulation 121:948-954. https://doi.org/10. 1161/CIRCULATIONAHA.109.192666

10. Dor-Haim H, Barak S, Horowitz M, Yaakobi E, Katzburg S, Swissa M, Lotan C (2018) Improvement in cardiac dysfunction with a novel circuit training method combining simultaneous aerobic-resistance exercises. A randomized trial. PLoS ONE 13(1):e0188551. https://doi.org/10.1371/journal. pone. 0188551

11. Ades PA (2001) Cardiac rehabilitation and secondary prevention of coronary heart disease. N Engl J Med 345(12):892-902

12. Leon AS, Franklin BA, Costa F, Balady GJ, Berra KA, Stewart KJ, Thompson PD, Williams MA, Lauer MS; American Heart Association; Council on Clinical Cardiology (Subcommittee on Exercise, Cardiac Rehabilitation, and Prevention); Council on Nutrition, Physical Activity, and Metabolism (Subcommittee on Physical Activity); American association of Cardiovascular and Pulmonary Rehabilitation (2005) Cardiac rehabilitation and secondary prevention of coronary heart disease: an American Heart Association scientific statement from the Council on Clinical Cardiology (Subcommittee on Exercise, Cardiac Rehabilitation, and Prevention) and the Council on Nutrition, Physical Activity, and Metabolism (Subcommittee on Physical Activity), in collaboration with the American association of Cardiovascular and Pulmonary Rehabilitation. Circulation 111(3):369376. Erratum in: Circulation. 2005;111(13):1717.

13. Piepoli MF, Corrà U, Benzer W, Bjarnason-Wehrens B, Dendale P, Gaita D, McGee H, Mendes M, Niebauer J, Zwisler AD, Schmid JP (2010) Secondary prevention through cardiac rehabilitation: from knowledge to implementation. A position paper from the Cardiac Rehabilitation Section of the European Association of Cardiovascular Prevention and Rehabilitation. Eur J Cardiovasc Prev Rehabil 17:1-17

14. Graham I, Atar D, Borch-Johnsen K, Boysen G, Burell G, Cifkova R, Dallongeville J, De Backer G, Ebrahim S, Gjelsvik B, Herrmann-Lingen C, Hoes A, Humphries S, Knapton M, Perk J, Priori SG, Pyorala K, Reiner Z, Ruilope L, Sans-Menendez S, Op Reimer WS, Weissberg P, Wood D, Yarnell J, Zamorano JL, Walma E, Fitzgerald T, Cooney MT, Dudina A, Vahanian A, Camm J, De Caterina R, Dean V, Dickstein K, Funck-Brentano C, Filippatos G, Hellemans I, Kristensen SD, McGregor K, Sechtem U, Silber S, Tendera M, Widimsky P, Zamorano JL, Altiner A, Bonora E, Durrington PN, Fagard R, Giampaoli S, Hemingway H, Hakansson J, Kjeldsen SE, Larsen mL, Mancia G, Manolis AJ, Orth-Gomer K, Pedersen T, Rayner M, Ryden L, Sammut M, Schneiderman N, Stalenhoef AF, Tokgözoglu L, Wiklund O, Zampelas A; European Society of Cardiology (ESC); European Association for Cardiovascular Prevention and Rehabilitation (EACPR); Council on CardiovascularNursing; European Association for Study of Diabetes (EASD); International Diabetes Federation Europe (IDF-Europe); European Stroke Initiative (EUSI); Society of Behavioural Medicine (ISBM); European Society of Hypertension (ESH); WONCA Europe (European Society of General Practice/Family Medicine); European Heart Network (EHN); European Atherosclerosis Society (EAS) (2007) European guidelines on cardiovascular disease prevention in clinical practice: full text: fourth Joint Task Force of the European Society of Cardiology and other Societies on Cardiovascular Disease Prevention in Clinical Practice (constituted by representatives of nine societies and by invited experts). Eur J Cardiovasc Prev Rehabil 14(Supp 2):S1-S113

15. Giannuzzi P, Mezzani A, Saner H, Björnstad H, Fioretti P, Mendes M, CohenSolal A, Dugmore L, Hambrecht R, Hellemans I, McGee H, Perk J, Vanhees L, Veress G, Working Group on Cardiac Rehabilitation and Exercise Physiology. European Society of Cardiology (2003) Physical activity for primary and secondary prevention. Position paper of the Working Group on Cardiac Rehabilitation and Exercise Physiology of the European Society of Cardiology. Eur J Cardiovasc Prev Rehabil 10:319-327

16. Bjarnason-Wehrens B, Mayer-Berger W, Meister ER, Baum K, Hambrecht $\mathrm{R}$, Gielen S (2004) Recommendations for resistance exercise in cardiac rehabilitation. Recommendations of the German Federation for Cardiovascular Prevention and Rehabilitation. Eur J Cardiovasc Prev Rehabil 11(4):352-361

17. Beckers PJ, Denollet J, Possemiers NM, Wuyts FL, Vrints CJ, Conraads VM (2008) Combined endurance-resistance training vs. endurance training in patients with chronic heart failure: a prospective randomized study. Eur Heart J 29:1858-1866

18. Braith RW, Beck DT (2008) Resistance exercise: training adaptations and developing a safe exercise prescription. Heart Fail Rev 13:69-79

19. American College of Sports Medicine (2009) Guidelines for exercise testing and prescription, 8th edn. Lippincott Williams \& Wilkins, Philadelphia

20. Selig SE, Carey MF, Menzies DG, Patterson J, Geerling RH, Williams AD, Bamroongsuk V, Toia D, Krum H, Hare DL (2004) Moderate-intensity resistance exercise training in patients with chronic heart failure improves strength, endurance, heart rate variability, and forearm blood flow. J Card Fail 10:21-30

21. Ades PA, Savage PD, Cress ME, Brochu M, Lee NM, PoehIman ET (2003) Resistance training on physical performance in disabled older female cardiac patients. Med Sci Sports Exerc 35:1265-1270

22. Brochu M, Savage P, Lee M, Dee J, Cress ME, Poehlman ET, Tischler M, Ades PA (2002) Effects of resistance training on physical function in older disabled women with coronary heart disease. J Appl Physiol 92:672-678

23. Marzolini S, Oh Pl, Thomas SG, Goodman JM (2008) Aerobic and resistance training in coronary disease: single versus multiple sets. Med Sci Sports Exerc 40:1557-1564

24. Santa-Clara H, Fernhall B, Mendes M, Sardinha LB (2002) Effect of a 1 year combined aerobic- and weight-training exercise program on aerobic capacity and ventilatory threshold in patients suffering from coronary artery disease. Eur J Appl Physiol 87:568-575

25. Kida K, Osada N, Akashi YJ, Sekizuka H, Omiya K, Miyake F (2008) The exercise training effects of skeletal muscle strength and muscle volume to improve functional capacity in patients with myocardial infarction. Int J Cardiol 129:180-186

26. Schmid JP, Anderegg M, Romanens M, Morger C, Noveanu M, Hellige G, Saner H (2008) Combined endurance/resistance training early on, after a first myocardial infarction, does not induce negative left ventricular remodelling. Eur J Cardiovasc Prev Rehabil 15:341-346

27. Arthur HM, Gunn E, Thorpe KE, Ginis KM, Mataseje L, McCartney N, McKelvie RS (2007) Effect of aerobic vs combined aerobic-strength training on 1-year, post-cardiac rehabilitation outcomes in women after a cardiac event. J Rehabil Med 39:730-735

28. Degache F, Garet M, Calmels P, Costes F, Bathelemy JC, Roche F (2007) Enhancement of isokinetic muscle strength with a combined training program in chronic heart failure. Clin Physiol Funct Imaging 27:225-230

29. Izawa KP, Watanabe S, Osada N, Kasahara Y, Yokoyama H, Hiraki K, Morio Y, Yoshioka S, Oka K, Omiya K (2009) Handgrip strength as a predictor of prognosis in Japanese patients with congestive heart failure. Eur J Cardiovasc Prev Rehabil 16:21-27

30. Spruit MA, Eterman RM, Hellwig V, Janssen P, Wouters E, Uszko-Lencer $N$ (2009) A systematic review on the effects of moderate- to-high intensity resistance training in patients with chronic heart failure. Heart 95:1399-1408

31. Ehlke K, Greenwood M (2006) Resistance exercise for post-myocardial infarction patients: current guidelines and future considerations. Strength Cond J 28:56-62

32. Hansen D, Dendale P, Berger J, Meeusen R (2005) Rehabilitation in cardiac patients: what do we know about training modalities? Sports Med 35:1063-1084

33. Currie KD, Bailey KJ, Jung ME, McKelvie RS, MacDonald MJ (2015) Effects of resistance training combined with moderate-intensity endurance or low-volume high-intensity interval exercise on cardiovascular risk factors in patients with coronary artery disease. J Sci Med Sport 18(6):637-642. https://doi.org/10.1016/j.jsams.2014.09.013

34. Berent $R$, von Duvillard SP, Crouse SF, Sinzinger $H$, Green JS, Schmid P (2011) Resistance training dose response in combined endurance-resistance training in patients with cardiovascular disease: a randomized trial. Arch Phys Med Rehabil 92:1527-1533

35. Moher D, Liberati A, Tetzlaff J, Altman DG (2009) The PRISMA Group preferred reporting items for systematic reviews and meta-analyses: the PRISMA Statement. PLoS Med 6(7):e1000097. https://doi.org/10.1371/ journal.pmed. 1000097

36. PEDro. https://www.pedro.org.au/

37. Marzolini S, Oh PI, Brook D (2011) Effect of combined aerobic and resistance training versus aerobic training alone in individuals with coronary artery disease: a meta-analysis. Eur J Prev Cardiol 19(1):81-94. https://doi. org/10.1177/1741826710393197 
38. Caruso FR, Arena R, Phillips SA, Bonjorno JC Jr, Mendes RG, Arakelian VM, Bassi D, Nogi C, Borghi-Silva A (2015) Resistance exercise training improves heart rate variability and muscle performance: a randomized controlled trial in coronary artery disease patients. Eur J Phys Rehabil Med 51:281-289

39. Gremeaux Pole V, Duclay J, Deley G, Philipp JL, Laroche D, Pousson M, Casillas Pole JM (2010) Does eccentric endurance training improve walking capacity in patients with coronary artery disease? A randomized controlled pilot study. Clin Rehabil 24:590-599

40. Guiraud T, Labrunée M, Besnier F, Sénard J, Pillard F, Riviére D, Richard L, Laroche D, Sanguignol F, Pathak A, Gayda M, Gremeaux V (2017) Wholebody strength training with Huber Motion Lab and traditional strength training in cardiac rehabilitation: a randomized controlled study. Ann Phys Rehabil Med 60:20-26

41. Yamamoto S, Matsunaga A, Kamiya K, Miida K, Ebina Y, Hotta K, Shimizu R, Matsuzawa R, Abe Y, Kimura M, Shimizu S, Watanabe H, Noda C, Yamaoka-Tojo M, Masuda T, Izumi T (2012) Walking speed in patients with first acute myocardial infarction who participated in a supervised cardiac rehabilitation program after coronary intervention. Int Heart J 53:347-352

42. Kamiya K, Mezzani A, Hotta K, Shimizu R, Kamekawa D, Noda C, YamaokaTojo M, Matsunaga A, Masuda T (2014) Quadriceps isometric strength as a predictor of exercise capacity in coronary artery disease patients. Eur J Prev Cardiol 21:1285-1291

43. Lamotte M, Niset G, van de Borne P (2005) The effect of different intensity modalities of resistance training on beat-to-beat blood pressure in cardiac patients. Eur J Cardiovasc Prev Rehabil 12:12-17

44. Wenger NK, Froelicher ES, Smith LK, Ades PA, Berra K, Blumenthal JA, Certo CM, Dattilo AM, Davis D, DeBusk RF et al (1995) Cardiac rehabilitation as secondary prevention Agency for Health Care Policy and Research and National Heart, Lung, and Blood Institute. Clin Pract Guidel Quick Ref Guide Clin 17:1-23

45. Fragnoli-Munn K, Savage PD, Ades PA (1998) Combined resistive aerobic training in older patients with coronary artery disease early after myocardial infarction. J Cardiopulm Rehabil 18:416-420

46. Pu CT, Johnson MT, Forman DE et al (2001) Randomized trial of progressive resistance training to counteract the myopathy of chronic heart failure. J Appl Physiol 90:2341-2350

47. Pollock ML, Franklin BA, Balady GJ, Chaitman BL, Fleg JL, Fletcher B, Limacher M, Piña IL, Stein RA, Williams M, Bazzarre T, AHA Science Advisory (2000) Resistance exercise in individuals with and without cardiovascular disease: benefits, rationale, safety, and prescription: an advisory from the Committee on Exercise, Rehabilitation, and Prevention, Council on Clinical Cardiology, American Heart Association. Circulation 10:828-833

48. Warburton DER, Gledhill N, Quinney A (2001) Musculoskeletal fitness and health. Can J Appl Physiol 26:217-237

49. Williams MA, Haskell WL, Ades PA, Amsterdam EA, Bittner V, Franklin BA, Gulanick M, Laing ST, Stewart KJ, American Heart Association Council on Clinical Cardiology; American Heart Association Council on Nutrition, Physical Activity, and Metabolism (2007) Resistance exercise in individuals with and without cardiovascular disease: 2007 update. Circulation 116(5):572-584

50. Pratley R, Nicklas B, Rubin M (1994) Strength training increases resting metabolic rate and norepinephrine levels in healthy 50- to 65-year-old men. J Appl Physiol 76:133-137

51. Despres JP, Moorjani S, Lupien PJ, Tremblay A, Nadeau A, Bouchard C (1990) Regional distribution of body fat, plasma lipoproteins, and cardiovascular disease. Arteriosclerosis 10:497-511

52. Marzolini S, Swardfager W, Alter DA, Oh PI, Tan Y, Goodman JM (2015) Quality of life and psychosocial measures influenced by exercise modality in patients with coronary artery disease. Eur J Phys Rehabil Med 51(3):291-299

\section{Publisher's Note}

Springer Nature remains neutral with regard to jurisdictional claims in published maps and institutional affiliations.

\section{Submit your manuscript to a SpringerOpen ${ }^{\circ}$ journal and benefit from:}

- Convenient online submission

- Rigorous peer review

- Open access: articles freely available online

- High visibility within the field

- Retaining the copyright to your article

Submit your next manuscript at $\boldsymbol{\nabla}$ springeropen.com 